\title{
Tratamiento médico de la enfermedad hidatídica
}

\author{
Pedro Pablo Pinto G.
}

\begin{abstract}
RESUMEN
Se presenta una puesta al día sobre el tratamiento médico de la enfermedad hidatídica, poniendo especial énfasis en sus indicaciones del pre, postoperatorio y en el manejo de la enfermedad hidatídica propiamente tal. Además se analiza el mecanismo de acción, farmacocinética, pautas de administración $y$ efectos adversos tanto de los benzoimidazoles como de las isoquinoleinas, puesto que ambos quimioterápicos son de uso común en esta patología. (Palabras claves/Key words: Enfermedad hidatídica/ Hydatid disease;Tratamiento médico/ Medical treatment).
\end{abstract}

\section{INTRODUCCIÓN}

Sin desconocer que la cirugía sigue siendo el tratamiento de elección en la enfermedad hidatídica, hay situaciones que son importantes de tener presente:

- Aún en los mejores centros, la mortalidad quirúrgica fluctúa entre el 0.9 y $3.6 \%{ }^{1}$.

- El pronóstico empeora en la segunda operación, ya que la mortalidad aumenta a un $6 \%$ y a un $20 \%$ en la tercera ${ }^{2}$.

- La morbilidad postoperatoria alcanza a cifras de hasta $57,1 \% 3$.

- La recidiva de la enfermedad (debido a su ruptura espontánea o traumática, filtración durante el acto operatorio o debido a su incompleta extirpación), fluctúa entre un 10 y $30 \%$ dependiendo del período de seguimiento ${ }^{4,5}$.

- La negativa de los pacientes a someterse a cirugía.

Todo lo anterior ha motivado la búsqueda de alternativas médicas, que combinadas o no con la cirugía, disminuyan de algún modo estas negativas estadísticas.

El tratamiento médico se inicia en 1977 con la publicación de los cuatro primeros casos tratados exitosamente con Mebendazol; desde ese entonces hasta ahora hay más de 1500 casos documentados y controlados ${ }^{6}$.
A continuación, nos referimos al mecanismo de acción, farmacocinética y pautas de administración, tanto de los benzoimidazoles como de las isoquinoleinas.

\section{MECANISMO DE ACCION}

Los benzoimidazoles centran su acción a nivel de la membrana germinativa del parásito, provocando alteraciones funcionales y morfológicas, que se traducen en modificación de la presión osmótica, reducción del tamaño y finalmente la muerte del quiste.

El mecanismo íntimo de actuación es mediante la inhibición de la tubulina, obstaculizando con ello la absorción de la glucosa, lo que provoca una depleción del glicógeno y secundariamente alteraciones degenerativas del retículo endoplásmico y mitocondrias de la capa germinativa del quiste ${ }^{7,8}$.

Las isoquinoleinas producen una rápida contracción del parásito debido a un aumento de la permeabilidad de la membrana celular al calcio. Esto provoca una contractura y parálisis de la musculatura parasitaria, lo que favorece su expulsión del tejido parasitario.

\section{FARMACOCINÉTICA}

\section{A. Benzoimidazoles}

El Mebendazol es insoluble en agua, éter, alcohol y cloroformo, lo que determina un bajo 
nivel de absorción intestinal, $20 \%$, lo cual obliga a emplear altas dosis para conseguir niveles plasmáticos óptimos ${ }^{10}$.

La absorción del Albendazol es mayor, pues aunque es insoluble en agua sí lo es en solventes orgánicos. Se ha visto que el principio activo es uno de sus metabolitos, el Albendazol Sulfóxido, cuya concentración plasmática es 10 veces superior a la del Mebendazol ${ }^{10}$.

Los niveles plasmáticos de los benzoimidazoles oscilan entre un $5-10 \%$ de la dosis ingerida. Sin embargo, las concentraciones intraquísticas corresponden al $10 \%$ del nivel plasmático en el caso del Mebendazol y al $20 \%$ en el caso del Albendazol ${ }^{11}$.

\section{B. Isoquinoleinas}

El Praziquantel es rápidamente absorbido después de su administración oral y es metabolizado y eliminado por los riñones.

En el plasma alcanza altas concentraciones del orden de 1,3-1,8 mg/lt, con dosis de $50 \mathrm{mg} /$ $\mathrm{kg}$, ya sea dándose diariamente, semanalmente, quincenalmente o mensualmente ${ }^{12}$.

Presenta un efecto 10 veces mayor que el Albendazol, tanto es así, que concentraciones tan bajas de Praziquantel como $20 \mathrm{mg} / \mathrm{lt}$ son equivalentes a $250 \mathrm{mg} / \mathrm{lt}$ de Albendazol ${ }^{13}$. Lamentablemente estudios in vivo sobre su uso en la echinococcosis secundaria no han sido concluyentes. Lo que sí se ha visto, es que en combinación con Albendazol potencia el efecto de este último en caso de filtración de contenido quístico ${ }^{14}$.

La mayor parte de estos fármacos se eliminan entre 10 y 24 horas por el tubo digestivo sin sufrir alteraciones. El pequeño porcentaje absorbido es rápidamente metabolizado y excretado por la orina en un plazo de 24-48 horas, en forma de fármacos inalterados o bien como derivados descarboxilados ${ }^{7}$.

\section{PAUTAS DE ADMINISTRACIÓN}

La forma de administración es vía oral

- Mebendazol: 50-70 mg/kg/día, repartido en tres dosis durante el día ${ }^{14}$

- $\quad$ Albendazol: $10-15 \mathrm{mg} / \mathrm{kg} / \mathrm{día}$, repartido en dos dosis a lo largo del día, acompañado de 14 días de descanso entre cada ciclo ${ }^{15}$. Sin embargo, últimamente se han realizado tratamientos continuos con mejores resultados y sin incrementar los efectos adversos ${ }^{16}$
- Praziquantel: 25-50 mg/kg ya sea una vez al día, o una vez a la semana o cada 15 días ${ }^{9}$.

La absorción de los benzoimidazoles y la isoquinoleinas se ve aumentada con el uso de Cimetidina ${ }^{17}$ y con la administración de comidas ricas en grasa $^{18}$.

\section{EFECTOS ADVERSOS}

\section{a. Benzoimidazoles}

- Cambio en los niveles enzimáticos hepáticos. Aproximadamente $10-20 \%$ de los pacientes desarrollan aumento de las transaminasas en algún momento durante el tratamiento. Esta alza es suave, moderada, autolimitada y reversible con la suspensión del tratamiento. El incremento de las transaminasas se correlaciona con la efectividad de la terapia y con la temprana ocurrencia de modificaciones degenerativas del quiste ${ }^{16}$. La causa de esta elevación estaría dada por la inflamación de la periquística, secundaria a la reacción inmune del huésped, lo que se traduciría en liberación de material tóxico por parte del hígado. Es indudable que tampoco se puede descartar una hepatotoxicidad directa del medicamento. En la serie europea sólo un $3.8 \%$ de los pacientes debió suspender el tratamiento por anormalidades enzimáticas ${ }^{6}$. Aplasia medular de causa desconocida. Probablemente está relacionada a los benzoimidazoles. Excepcionalmente se manifiesta con una severa pancitopenia, agranulositosis o neutropenia. Se han descrito 2 muertos por esta causa con el uso del Albendazol ${ }^{6}$.

- Alopecia. Es más frecuente, $2.8 \%$, pero menos grave y se recupera al suspender el tratamiento ${ }^{6}$.

\section{b. Isoquinoleinas}

El Praziquantel es generalmente bien tolerado, los efectos adversos son usualmente suaves y transitorios. Nauseas, vómitos, dolor abdominal y vértigo han sido asociados a esta droga ${ }^{9}$.

\section{INDICACIONES DEL TRATAMIENTO MEDICO}

Tratamiento preoperatorio cuyos objetivos son:

- Reducir la presión intraquística y facilitar su remoción. 
- Disminuir el número de protoescolex vivos.

- Disminuir el riesgo de siembra en caso de filtración.

La duración de la terapia no se ha definido claramente, pero se sugiere que en quistes pequeños, menores de $5 \mathrm{~cm}$, de pared delgada, univesiculares, el tratamiento sea de 6 semanas ${ }^{1}$. En quistes grandes, mayores de $5 \mathrm{~cm}$, de pared gruesa, multivesiculares, la terapia debe prolongarse a 8 semanas ${ }^{1}$.

Tratamiento postoperatorio está orientado a:

- Evitar o disminuir el crecimiento del quiste en caso de filtración.

- Destruir quistes pequeños que hayan pasado inadvertidos durante el acto quirúrgico.

- Combatir la vesiculación exógena cuando no se ha realizado cirugía radical.

Se sugiere un tratamiento no menor de 8 semanas $^{7}$ e idealmente asociado a Praziquantel a dosis de $40 \mathrm{mg} / \mathrm{kg}$ una vez a la semana?

\section{TRATAMIENTO DE LA ENFERMEDAD HIDATÍDICA}

Es en este rubro donde se concentra la gran masa de publicaciones. La gran mayoría adolece de escasa representatividad y poca uniformidad en la dosis y duración de la terapia. Es por ello que trabajos realizados por la OMS ${ }^{19}$, Horton ${ }^{6}$, Teggi ${ }^{8}$ y Todorov ${ }^{15}$ entre otros, tratan de fijar normas en el manejo médico de la enfermedad hidatídica.

El mayor problema mencionado por muchos autores, es la falta de medios confiables para definir y medir la respuesta terapéutica. Es por ello, que con el desarrollo de los métodos no invasivos como la radiología, sonografía y tomografía computada, se ha logrado obtener las bases morfológicas que determinan el éxito o el fracaso de esta terapia medicamentosa. Los elementos a considerar son los siguientes ${ }^{7}$ :

- Alteración en la morfología del quiste.

- $\quad$ Alteración en la estructura intraquística.

- Cambio en la pared del quiste.

- Disminución del tamaño y deformación del quiste.

- Desaparición del quiste.

Los resultados se evalúan de acuerdo a la respuesta clínica y quística del quimioterápico, de acuerdo a pautas entregadas por la $\mathrm{OMS}^{20}$.

\section{Respuesta a nivel del quiste:}

- Cura. Cuando se produce completa desaparición del quiste.

- Mejoría. Cuando hay una reducción del volumen quístico mayor o igual al $25 \%$.

- Sin cambios. Cuando la variación de tamaño es menor del $25 \%$ y no hay variación en su morfología.

- Malo. Cuando hay un incremento en el tamaño o aparición de nuevos quistes.

\section{Respuesta clínica:}

- Cura. Aquellos pacientes que tienen más del $50 \%$ de sus quistes curados o bien que el $75 \%$ de ellos muestran alguna mejoría.

- Mejoría. Aquellos que presentan más del $25 \%$ de los quistes curados o la mejoría del $50-75 \%$ de ellos.

- $\quad$ Sin cambios. Aquellos que no presenten ninguna de las características anteriores.

En 1997 Horton $^{6}$ revisó la experiencia mundial tras 12 años de uso del Albendazol en la enfermedad hidatídica (1983 - 1995). De los 3.532 pacientes tratados sólo el 38\% fue evaluable, 1.369 pacientes (Tablas 1 y 2).

Tabla 1. Respuesta clínica al Albendazol ${ }^{6}$

\begin{tabular}{|lcccrc|}
\hline Fuente datos & $\mathbf{N}^{\circ}$ pacientes & Curados & Mejorados & Sin cambio & Malo \\
\hline Datos europeos & 253 & $72(28.5 \%)$ & $129(51.0 \%)$ & $46(18.1 \%)$ & $6(2.4 \%)$ \\
Publicaciones & 1.116 & $372(33.3 \%)$ & $469(42.0 \%)$ & $275(24.6 \%)$ & \\
Total & 1.369 & $444(32.4 \%)$ & $598(43.7 \%)$ & $327(23.9 \%)$ & \\
\hline
\end{tabular}

Tabla 2. Respuesta quística al Albendazol ${ }^{6}$

\begin{tabular}{|lccrrr|}
\hline Fuente datos & Quistes evaluables & Cura & Mejoría & Sin cambio & Malo \\
\hline Datos europeos & 435 & $160(35.2 \%)$ & $187(41.1 \%)$ & $102(22.4 \%)$ & $6(1.3 \%)$ \\
Publicaciones & 2912 & $663(22.8 \%)$ & $1.418(48.7 \%)$ & $83(28.5 \%)$ & \\
Total & 3347 & $823(24.6 \%)$ & $1.605(48 \%)$ & & \\
\hline
\end{tabular}


Se concluye por lo tanto que:

$30 \%$ de los pacientes presentan desaparición de los quistes (Cura). $40-50 \%$ hay significativa reducción o degeneración quística (Mejoría).

$20-30 \%$ no hay cambios morfológicos (Fracaso).

\section{CONSIDERACIONES GENERALES RESPECTO AL TRATAMIENTO}

a. La quimioterapia es más efectiva $e^{21}$ :

- Pacientes menores de 30 años, pues los quistes presentan paredes más delgadas.

- Quistes menores de $5 \mathrm{~cm}$.

- Quistes múltiples (sobre 10 quistes).

- Quistes ubicados en hígado, pulmón y peritoneo ${ }^{6}$.

b. La quimioterapia es menos efectiva en ${ }^{20}$ :

- Quistes viejos en pacientes mayores de 30 años.

- Quistes multivesiculares mayores de 10 $\mathrm{cm}$.

- Quistes óseos ${ }^{19}$.

\section{DURACIÓN DE LA TERAPIA}

En general las grandes series realizan 3 ciclos de 28-30 días, seguido de 14 días de descanso. Se ha visto que con menos de 3 ciclos responde sólo el $59 \%$, comparado con el $74 \%$ cuando se realizan entre $3-5$ ciclos y del $83 \%$ cuando se realizan más de seis ${ }^{16}$. Por lo tanto se aconseja realizar rutinariamente 3 ciclos y muy excepcionalmente más de seis $^{23}$.

\section{RECURRENCIA}

Series con más de 5 años de seguimiento demuestran que las recidivas varían del 3 al $30 \%{ }^{17}$. Un estudio realizado por Franchi ${ }^{23}$ (mayor serie personal publicada, 442 pacientes y 882 quistes) señala que las $3 / 4$ partes de las recidivas se presentaron dentro de los 2 años de finalizada la terapia y que los quistes hepáticos recayeron más frecuentemente que los pulmonares, 33.9 contra $9.7 \%$.

Estas recurrencias al ser retratadas responden en el $95 \%{ }^{16}$.

\section{TRATAMIENTO MEDICO DE LA HIDATIDOSIS PULMONAR}

Desde la introducción de los benzoimidazoles en los años 70 hay sólo 100 casos documentados y controlados de quistes hidatídicos pulmonares tratados en forma médica ${ }^{20,21,23-25}$.

Hay aspectos interesantes que requieren de un comentario especial:

- Indicación de la terapia. Se aconseja realizarlo en pacientes con hidatidosis pulmonar múltiple e hidatidosis pulmonar única, con quistes menores de $5 \mathrm{~cm}$ y univesiculares. Se ha visto que la mejor respuesta se encuentra en quistes de $3-5$ $\mathrm{cm}$ con un $71.7 \%$ de resultados positivos ${ }^{26}$. No se aconseja en quistes multivesiculares por su mala respuesta ${ }^{24}$.

- Elección del quimioterápico. Se recomienda el Albendazol por presentar una mejor absorción y por ende una mejor concentración a nivel del quiste. Franchi concluye que los cambios degenerativos en los quistes con el Albendazol fue de un $82.2 \%$ contra un $56.1 \%$ del Mebendazol, lo cual fue estadísticamente significativo ${ }^{23}$.

- Dosis: $10 \mathrm{mg} / \mathrm{kg} / \mathrm{día}$ con descanso de 14 días.

- Duración. Como se ha mencionado, se requiere una duración mayor de un mes. En nuestra casuística realizamos 2 ciclos $^{26}$. Al revisar la literatura se menciona duración de 2-5 ciclos, ya sea en forma continua, o con descanso de 15 días entre cada ciclo ${ }^{20,21,23-25 .}$

- Resultados. De acuerdo a la revisión de las publicaciones y de nuestros pacientes hubo $20-26$ :

- Desaparición del quiste $\quad: 35 \%$

- Disminución de tamaño : $: 24.6 \%$

- Sin variación : :33.8\%

- Mala respuesta $\quad: 7 \%$

La forma más habitual de resolución del quiste pulmonar fue la ruptura y posterior vómica, 48-61.5\% ${ }^{20-21}$. Es importante mencionar que en el $57 \%$ los quistes desaparecen durante los tres primeros meses de terminada la terapia y que el $30 \%$ lo hace después del año; motivo por el cual el seguimiento es fundamental ${ }^{26}$. 


\section{REFERENCIAS}

1. Horton RJ: Chemotherapy of echinococcus infection in man with albendazol. Trans Roy Soc Trop Med Hyg 1989; 83: 97-102

2. Amir Jahed AK, Fardin R, Farzad A, Bakshandeh K: Clinical echinococcosis. Ann Surg 1975; 182: 541-6

3. Mottaghian H, Saidi F: Postoperative recurrence of hydatid disease. Br J Surg 1978; 65; 237-42

4. Schiller CF: Complication of echinococcus cyst rupture. JAMA 1966; 195: 158-60

5. Pinto P: Hidatidosis hepática: Estudio de una serie de 534 casos. Rev Chil Cir 1991; 43: 184-7

6. Horton RJ: Albendazole in treatment of human cystic echinococcosis: 12 years of experience. Acta Trop 1997; 64: 79-93

7. Pinto P: Tratamiento médico de la hidatidosis. Rev Chil Cir 1994; 46: 437-40

8. Teggi A, Lastilla MG, De Rosa F: Therapy of human hydatid disease with mebendazole and albendazole. Antimicrob Agents Chemother 1993; 37: 1679-84

9. Anadol D, Ozcelik U, Kiper N, Gocmen A: Treatment of hydatid disease. Paediatr Drugs 2001; 3: 123-35

10. Morris DL, Dykes PW, Marriner S, Bogan J, Burrows F, Skeene-Smith $\mathrm{H}$ et al: Albendazole. Objetive evidence of responce in human hydatid disease. JAMA 1985; 253: 2053-7

11. Morris DL, Chinnery JB, Georgiou G, Stamatakis G, Golematis B: Penetration of albendazole sulphoxide into hydatid cyst. Gut 1987; 28: $75-80$

12. Mohamed AE, Yasawy MI, Al Karawi MA: Combined albendazole and praziquantel versus albendazole in the treatment of hydatid disease. Hepatogastroenterology 1998; 45: 1690-4

13. Taylor DH, Morris DL: Combination chemotherapy is more effective in post-spillage prophylaxis for hydatid disease than either albendazole or praziquantel alone. Br J Surg 1989; 76: 954

14. Cobo F, Yarnoz C, Sesma B, Fraile P, Aizcorbe $\mathrm{M}$, Trujillo $\mathrm{R}$ et al. Albendazole plus praziquantel versus albendazole alone as a preoperative treatment in intrabdominal hidatidosis caused by Echinococcus granulosus. Trop Med Int Health 1998; 3: 462-6.
15. Todorov T, Vutova K, Mechkov G, Tonchev Z, Georgiev P, Lazarova I: Experience in chemotherapy of severe, inoperable echinococcosis in man. Infection 1992; 20: 19-24

16. Saimot A: Medical treatment of liver hydatidosis. World J Surg 2001; 25: 15-20

17. Wen H, Zhang HW, Muhmut M, Zou PF, New RR, Craig PS: Initial observation on albendazole in combination with cimetidine for the treatment of human cystic echinococcosis. Ann Trop Med Parasitol 1994; 88: 49

18. Lange H, Eggers R, Bircher J: Increased systemic availability of albendazole when taken with a fatty meal. Eur J Clin Pharmacol 1988; 34: 315

19. Davis A, Dixon $H$, Pawlowski ZS: Multicentre clinical trials of benzimidazole carbamates in human cystic echinococcosis (phase 2). Bull World Health Organ 1989; 67: 503-8

20. Keshmiri M, Baharvahdat $\mathrm{H}$, Fattahi $\mathrm{SH}$, Davachi B, Dabiri $\mathrm{RH}$, Baradaran $\mathrm{H}$ et al: $\mathrm{A}$ placebo controlled study of Albendazole in the treatment of pulmonary echinococcosis. Eur Respir J 1999; 14: 503-07

21. Nahmias J, Goldsmith R, Soibelman M, el-On J: Three to 7 year follow up after albendazole treatment of 68 patients with cystic echinococcosis (hydatid disease). Ann Trop Med Parasitol 1994; 88: 295-04

22. WHO Informal Working Group on Echinococcosis: Guidelines for treatment of cystic and alveolar echinococcosis in humans. Bull World Health Organ 1996; 74: 231-42

23. Franchi C, Di Vico B, Teggi A: Long term valuation of patients with hydatidosis treated with bezimidazole carbamate. Clin Infect Dis 1999; 29: 304-9

24. Aggarwal P, Wali JP: Albendazole in the treatment of pulmonary echinococcosis. Thorax 1991; 46: 599-600

25. Todorov T, Mechkov G, Vutova K, Georgiev $\mathrm{P}$, Lazarova I, Tonchev $\mathrm{Z}$ et al. Factors influencing the responce to chemotherapy in human cystic echinococcosis. Bull WHO 1992; 70: 347-58

26. Pinto P, Ramesh T, Parra R: Albendazol en el tratamiento de la hidatidosis pulmonar. Rev Chil Cir 2002; 54: 265-8 